\title{
Dexmedetomidine: the first new kid on the block for preventing cardiac surgery- associated acute kidney injury?
}

\author{
Patrick M. Honore ${ }^{1 *}$, David De Bels ${ }^{1}$, Thierry Preseau ${ }^{2}$ and Herbert D. Spapen ${ }^{3}$ \\ See related letter by Shi and Tie https://ccforum.biomedcentral.com/articles/10.1186/s13054-017-1776-0
}

Cardiac surgery-associated acute kidney injury (CSA-AKI) affects up to $30 \%$ of patients undergoing cardiopulmonary bypass $(\mathrm{CPB})$ surgery and is the second most common cause of AKI in the intensive care unit $[1,2]$. To date, scarce evidence supports specific measures to prevent CSA-AKI. A recent meta-analysis by Shi and Tie [1] highlights the use of the selective $\alpha 2$-adrenoreceptor agonist dexmedetomidine (DEX) for prevention of CSA-AKI. The authors assume that this renoprotective effect is multifactorial, including both direct (by increasing renal blood flow (RBF) and diuresis) and indirect (by decreasing oxidative and inflammatory "stress") effects on the kidney [1].

The work of Shi and Tie must be interpreted with caution. Many cardiac surgery patients have pre-existent renal dysfunction or specific comorbidities that enhance the risk to develop AKI. Patients are also exposed to a compilation of per- and postoperative renal "aggressors", including the $\mathrm{CPB}$ procedure itself, aortic cross-clamping time, (poly)transfusion, vasopressor and inotropic treatment, and the use of particular colloid or crystalloid infusions. Also, long-standing venous congestion as a result of increased right ventricular afterload makes the kidneys more vulnerable to "congestive failure" in case of peri-operative hemodynamic instability [2]. Any baseline incongruity regarding these variables between DEX and controls may significantly undermine the value of a meta-analysis. We strongly argue against the idea that DEX provides kidney protection by simply improving RBF. An increase of RBF does not change renal tissue oxygenation but rather augments pre-glomerular arteriovenous oxygen shunting. Medullary hypoxic damage is the key responsible for CSA-AKI. Under normal conditions, the outer medulla already suffers borderline hypoxia and thus becomes

\footnotetext{
* Correspondence: Patrick.Honore@CHU-Brugmann.be

'ICU Department, Centre Hospitalier Universitaire Brugmann, Place Van

Gehuchtenplein,4, 1020 Brussels, Belgium

Full list of author information is available at the end of the article
}

particularly sensitive to prolonged or intermittent episodes of low oxygen supply caused by $\mathrm{CPB}$-induced renal vasoconstriction and hemodilution [3]. Moreover, on- and off-pump procedures cause capillary fall-out and capillary flow cessation, respectively, which may aggravate medullary hypoxia [4]. Of note is that propofol anesthesia was also found to significantly reduce incidence and severity of AKI in patients undergoing valvular heart surgery with CPB [5].

Taken together, a potential protective role of DEX in CSA-AKI can only be reliably appreciated when type and duration of surgery, patient characteristics and comorbidities, right heart hemodynamics, and peri-operative therapeutic strategies are taken into account. Furthermore, it requires comparison with other relevant sedatives and a more thorough insight into DEX-mediated effects on compartmental RBF distribution and renal microcirculation.

\section{Abbreviations \\ CPB: Cardiopulmonary bypass; CSA-AKI: Cardiac surgery-associated acute kidney injury; DEX: Dexmedetomidine; RBF: Renal blood flow}

\begin{abstract}
Authors' contributions
$\mathrm{PMH}$ and HDS designed the paper. $\mathrm{PMH}, \mathrm{DDB}$, TF, and HDS participated in drafting and reviewing. All authors read and approved the final version of the manuscript.
\end{abstract}

\section{Competing interests}

The authors declare that they have no competing interests.

\section{Publisher's Note}

Springer Nature remains neutral with regard to jurisdictional claims in published maps and institutional affiliations.

\section{Author details \\ ${ }^{1}$ ICU Department, Centre Hospitalier Universitaire Brugmann, Place Van Gehuchtenplein,4, 1020 Brussels, Belgium. ²Emergency Department, Centre Hospitalier Universitaire Brugmann, Brussels, Belgium. ${ }^{3}$ Universitair Ziekenhuis Brussel, VUB University, Brussels, Belgium.}


Received: 2 May 2018 Accepted: 17 May 2018

Published online: 05 June 2018

\section{References}

1. Shi R, Tie HT. Dexmedetomidine as a promising prevention strategy for cardiac surgery-associated acute kidney injury: a meta-analysis. Crit Care. 2017;21:198.

2. Gambardella I, Gaudino M, Ronco C, Lau C, Ivascu N, Girardi LN. Congestive kidney failure in cardiac surgery: the relationship between central venous pressure and acute kidney injury. Interact Cardiovasc Thorac Surg. 2016;23:800-5.

3. Lannemyr L, Bragadottir G, Krumbholz V, Redfors B, Sellgren J, Ricksten SE. Effects of cardiopulmonary bypass on renal perfusion, filtration, and oxygenation in patients undergoing cardiac surgery. Anesthesiology. 2017;126:205-13.

4. Atasever B, Boer C, Goedhart P, Biervliet J, Seyffert J, Speekenbrink R, Schwarte $L$, de Mol B, Ince C. Distinct alterations in sublingual microcirculatory blood flow and hemoglobin oxygenation in on-pump and off-pump coronary artery bypass graft surgery. J Cardiothorac Vasc Anesth. 2011;25:784-90.

5. Yoo YC, Shim JK, Song Y, Yang SY, Kwak YL. Anesthetics influence the incidence of acute kidney injury following valvular heart surgery. Kidney Int. 2014;86:414-22. 